\title{
Correction to: Anti-cyclic citrullinated peptide antibody in the cerebrospinal fluid in patients with rheumatoid arthritis who have central nervous system involvement
}

\author{
Misako Higashida-Konishi ${ }^{1}$ (D) Keisuke Izumi ${ }^{1,2}$ (D) Masako Tsukamoto ${ }^{1} \cdot$ Hiroaki Ohya $^{1} \cdot$ Nozomi Takasugi $^{1}$. \\ Satoshi Hama ${ }^{1} \cdot$ Yutaro Hayashi $^{1} \cdot$ Mari Ushikubo $^{1} \cdot$ Kumiko Akiya $^{1} \cdot$ Araki Kazuhiro $^{1} \cdot$ Yutaka Okano $^{1} \cdot$ Hisaji Oshima $^{1}$
}

Published online: 22 June 2020

(C) International League of Associations for Rheumatology (ILAR) 2020

\section{Correction to: Clinical Rheumatology}

$$
\text { https://doi.org/10.1007/s10067-020-05044-0 }
$$

The authors regret that the original published version of the above article contained errors. The authors requested that these be noted and that the original paper remains unchanged.

The authors' first affiliation should be presented as "Department of Connective Tissue Diseases, National Hospital Organization Tokyo Medical Center, 2-5-1 Higashigaoka, Meguro-ku, Tokyo, 152-8902 Japan".

The online version of the original article can be found at https://doi.org/ 10.1007/s10067-020-05044-0

\section{Keisuke Izumi}

izz@keio.jp

1 Department of Connective Tissue Diseases, National Hospital Organization Tokyo Medical Center, 2-5-1 Higashigaoka, Meguro-ku, Tokyo 152-8902, Japan

2 Division of Rheumatology, Department of Internal Medicine, Keio University School of Medicine, 35 Shinanomachi, Shinjuku-ku,

Tokyo 160-8582, Japan 
Figure 1 image was incorrect, and in Fig. 4 panel b, a typo error was present, "ANDs" should have been "AND". The corrected figures are now presented correctly as follows:

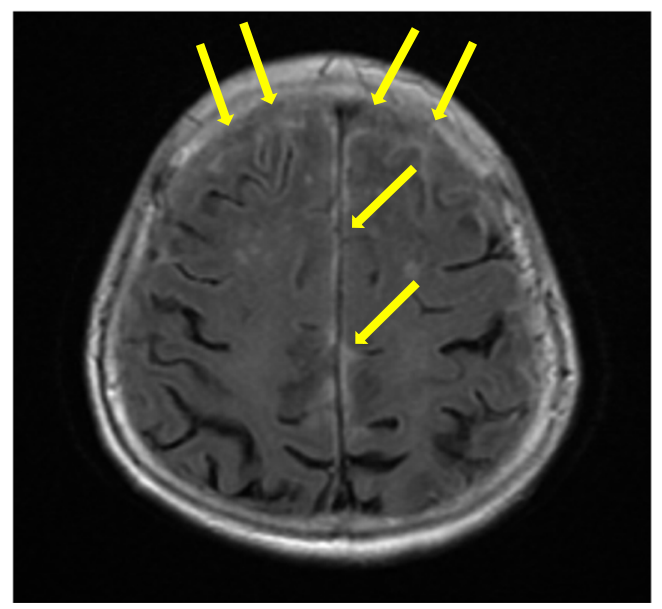

Fig. 1 T2 FLAIR MRI image, day 2. AT2 FLAIR MRI image showed a high signal in the subarachnoid space over the frontal and parietal lobes. FLAIR MRI, fluid-attenuated inversion recovery magnetic resonance imaging

Fig. 4 Flow diagram of the systematic literature review on patients with RA in whom ACPA in the CSF was measured. a Search term: "rheumatoid meningitis." b Search terms: "rheumatoid" AND ("leptomeningitis" OR "encephalitis" OR

"meningoencephalitis") AND "cerebrospinal fluid.” ACPA, anti-cyclic citrullinated peptide antibody; CSF, cerebrospinal fluid; RA, rheumatoid arthritis a) "rheumatoid meningitis" (Japan Medical Abstracts Society, PubMed, Medline)

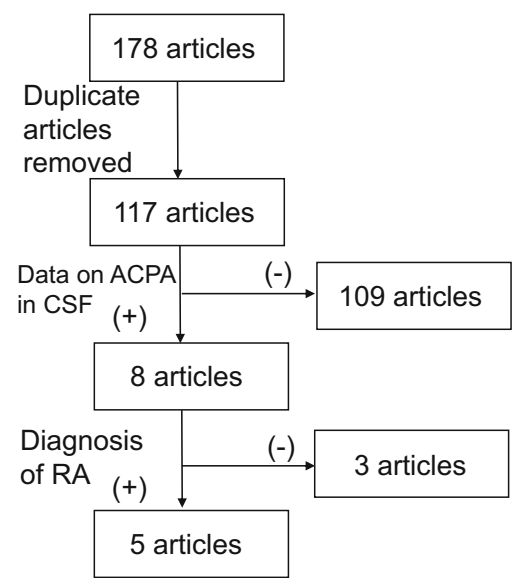

"rheumatoid" AND

("leptomeningitis" OR

b) "encephalitis" OR "meningoencephalitis") AND"cerebrospinal fluid" (Japan Medical Abstracts Society, PubMed, Medline)

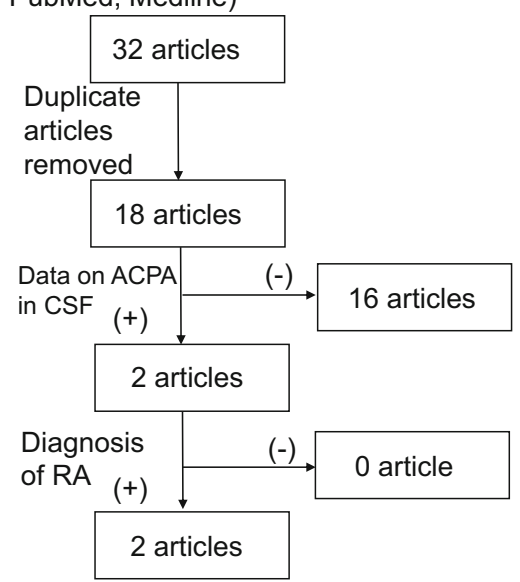

In the reference [15] of Table 1, the year "2014/2013" should have been " 2013 ". 


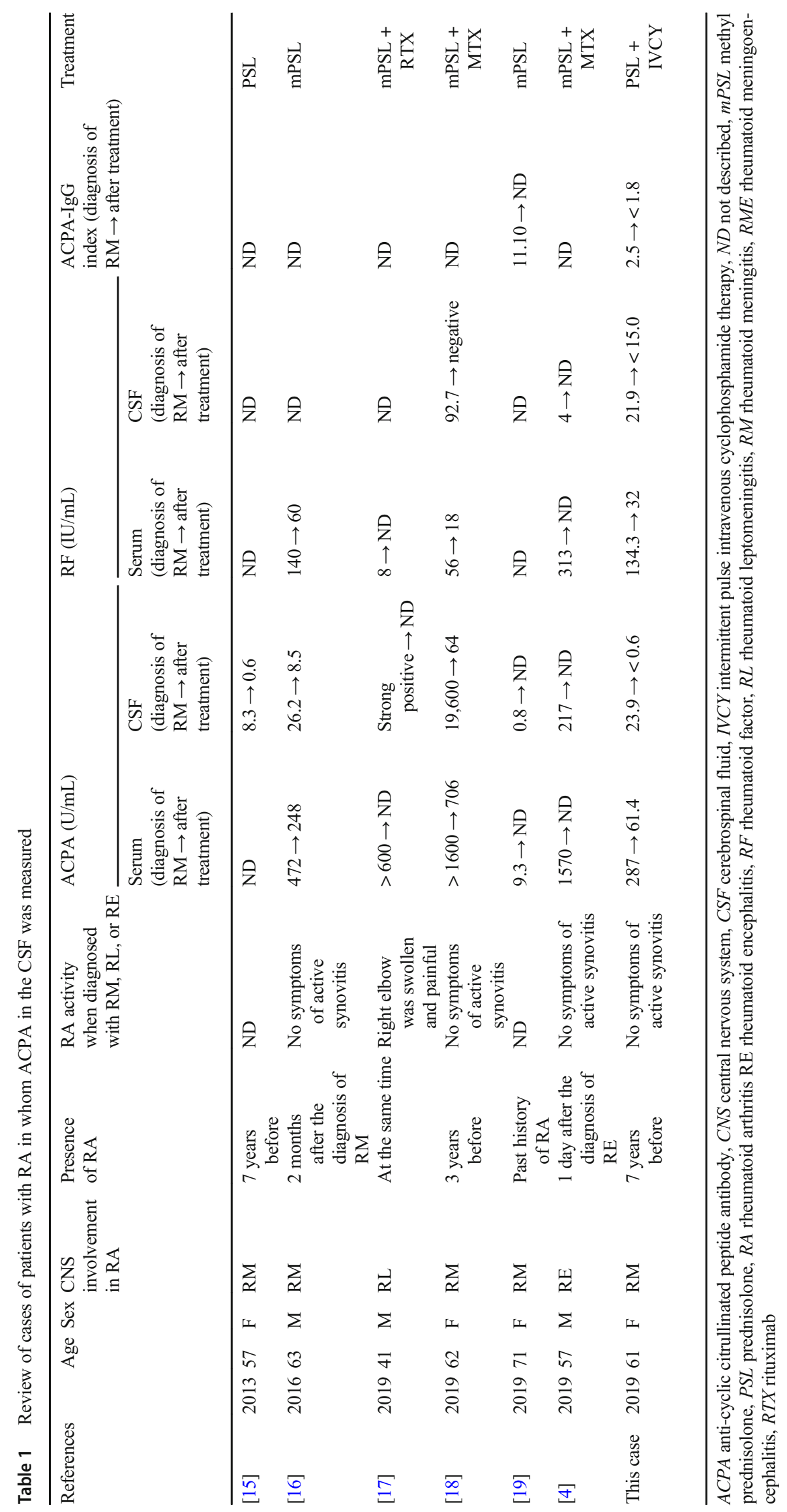




\section{References}

4. Kitamura E, Kondo Y, Kanazawa N, Akutsu T, Nishiyama K, lizuka T (2019) Autoimmune encephalitis as an extra-articular manifestation of rheumatoid arthritis. Intern Med 58:1007-1009

15. Ichiose S, Oohashi N, Watabe R, Satou S, Hoshi K, YahikoZawa H, Watanabe M (2013) A 57-year-old female case of rheumatoid meningitis with mental symptoms for which anti-cyclic citrullinated peptides antibody in the cerebrospinal fluid was useful for diagnosis. Med J Nagano Red Cross Hosp 27:110-111. (conference proceedings)

16. Shibahara T, Matsushita T, Matsuo R, Fukushima Y, Fukuda K, Sugimori H, Kamouchi M, Kitazono T, Ago T (2016) Anti-cyclic citrullinated peptide antibody positive meningoencephalitis in the preclinical period of rheumatoid arthritis. Case Rep Neurol 8:156160. https://doi.org/10.1159/000447627
17. Lubomski M, Sy J, Buckland M, Lee AS, Richards B, Thompson E, Fulham M, Breen N, Morris K, Halmagyi GM (2019) Rheumatoid leptomeningitis presenting with an acute neuropsychiatric disorder. Pract Neurol 19:68-71. https://doi.org/10.1136/ practneurol-2018-001978

18. Nissen MS, Nilsson AC, Forsberg J, Milthers J, Wirenfeldt M, Bonde C, Byg KE, Ellingsen T, Blaabjerg M (2019) Use of cerebrospinal fluid biomarkers in diagnosis and monitoring of rheumatoid meningitis. Front Neurol 10:666

19. Asano K, Kamimura N, Tsuchihashi Y, Watanabe D, Takahashi R (2019) A 71-year-old female with rheumatoid meningitis who presented with characteristic imaging findings on brain MRI and was useful for diagnosis by anti-CCP antibody in cerebrospinal fluid. Rinsho Shinkeigaku (Clinical Neurology) 59:299. (conference proceedings)

Publisher's note Springer Nature remains neutral with regard to jurisdictional claims in published maps and institutional affiliations. 